\title{
Smart Fingerprint Biometric and RFID Time-Based Attendance Management System
}

\author{
E.O. Badmus, O. P. Odekunle, and D. O. Oyewobi
}

\begin{abstract}
Attendance documentation is a crucial metric for determining a student's dedication, qualification, and status. Several types of systems have been developed for automated attendance. The majority of these implementations were built on a single factor template, representing a line of security error. In recent years, rapid development has taken place in several applications such as authorization, data access, and access control by implementing biometrics and radio frequency identification technologies. This research work focuses on designing a smart protocol for RFID authentication in combination with fingerprint biometric information, which guarantees confidentiality, identity verification, and data protection. Reaction time and event performance evaluation were the tests performed on the system. Ten students with unique fingerprints and RFID cards are assessed for the test. The test results show an average duration of 20.61 respective seconds for each student, and zero percent of false refusal rate was recorded in the implementation. Finally, the result shows the system is reliable and efficient. Each result revealed highspeed performance due to software and hardware coordination. Biometric systems provided data integrity and security, RFID provided limited complexity, and the proposed method's technical programming pattern provided an average run time.
\end{abstract}

Index Terms - Arduino, Biometrics, Fingerprint, RFID Technology, School Attendance.

\section{INTRODUCTION}

The action involving one being present, the pace at which a person is present, or the number of people in attendance are all examples of attendance [1]. There are a couple of attendance systems that have been implemented in this era. Attendance systems are being used to monitor employees' attendance and movement within their working hours in an establishment, students attending a lecture in an institution, people who participated in an event, and so on. Keeping track of attendance is an essential part of every institution's recordkeeping process. Researchers have developed different automated systems for the attendance-taking role [2]. Using fingerprint biometric technology combined with Radio Frequency Identification (RFID) would increase attendance records' reliability and credibility [3]. The fingerprint-RFIDbased attendance system speeds up taking attendance, reduces error, removes the traditional means of choosing the audience; this means all of it can be accomplished with minimum human contact and allows easier verification of involvement. This technology is being used in real-world

Submitted on June 17, 2021.

Published on July 27, 2021.

E. O. Badmus, University of Ibadan, Nigeria.

(corresponding e-mail: Emmanueloluwatobi81@gmail.com)

O. P. Odekunle, Federal University Oye-Ekiti, Nigeria.

(e-mail: preciousodekunle@gmail.com) applications such as attendance tracking in large companies, sectors, industries, universities, and secondary and primary schools, among others, where there are many participants. Within a fraction of a second, the system records people's involvement.

This system would aid in the generation of reports and the evaluation of a participant's attendance eligibility. RFID technology makes it simpler and faster to see what the participants are doing at any given time and reduces asset losses. For facilities management, fingerprint recognition is often used to recognize people more accurately and safely. The RFID-Fingerprint Attendance Management system is being built to provide a more safe, comfortable, and faster user authentication method than passwords and tokens.

\section{LITERATURE REVIEW}

Several systems have been developed for automated attendance. The majority of these systems in this review were built on a single factor template representing a security line of error compared to the proposed approach. Most of the attendance systems use paper-based methods for taking and calculating attendance and this manual method requires paper sheets and a lot of stationery material. Previously, very few works have been done relating to academic attendance monitoring problems.

The major works on automated attendance systems relating to biometrics and RFID by engineers and researchers in the past will be covered in this section. Fingerprint attendance systems and RFID-based attendance systems are examples of attendance systems. So, there are many ways of taking attendance electronically, which includes:

- Attendance system based on cards; examples are Smart cards, Proximity cards, Swipe cards, RFID cards, and tags.

- Attendance system based on something the user knows; examples are PIN and Password.

- The attendance system based on biometrics includes Fingerprint, Facial recognition, Hand geometry, Retina recognition, and Voice recognition.

This research work involved the use of cards and use of biometrics. As a result, engineers' and researchers' previous work on RFID cards and biometrics-based attendance systems will be examined.

Chiagozie and Nwaji [4] proposed an RFID-based attendance system with an automatic door unit. A radio

D. O. Oyewobi, Federal University Oye-Ekiti, Nigeria.

(email: danieloyewobi@gmail.com). 
frequency recognition system was set up as part of their work to create a time-attendance management system in the project. The microcontroller module and the radio frequency identification scanner made up the system. Compared to the conventional attendance scheme, it was noted that the project offers a convenient attendance method for performance and quality. As a result, the project could be implemented in both academic institutions and business areas. It was also noted that the author could enhance the attendance mechanism by incorporating a feature in which the attendance system could indicate when a worker or student is late for work or lectures, as the case may be. Consequently, the author could implement a biometric system to increase security.

Sabri et al, presented an intelligent attendance system by using RFID [5]. The author presented an intelligent attendance system design that used the information derived from the RFID database handling process. It was noted that to have complete system usability; one must develop an RFID database handling system. The intelligent attendance system had additional modules that helped lecturers and students in the classroom. Examples are in the form of distributing notes and reminders. The limitation of the attendance system was that it did not access enough appropriate data from the RFID server, which could help execute the attendance operation.

Eze et al, proposed a biometric-based attendance system with remote real-time monitoring for tertiary institutions in developing countries [6]. The authors presented the integrated Fingerprint Attendance Program with remote monitoring enablement for staff and students of tertiary institutions, which could be used for their work. This research work provided a web-based, real-time remote monitoring interface that allowed administrators to track staff and students' check-ins and check-out of the program. The program took approximately 2 seconds to check and record respective students' attendance instead of the usual 15 seconds duration for the manual processes. Seventy-one students were surveyed during the testing phase, in which it was noted that the program sped up attendance 7.3 times faster than the usual manual processes. It was then concluded that organization administrators (HODs, directors, deans, etc.) could track the attendance from anywhere in the world at any time.

Shoewu, Makanjuola, and Olatinwo discussed a biometricbased attendance system using the LASU Epe campus as a case study [7]. The authors proposed a biometrically dependent attendance program for course lectures. The proposed method automatically takes attendance using the student recognition process. The study achieved a 94\% success rate for the eight students that participated in the case study. It was noted that the biometric-based attendance system approximately averaged duration of 3.8 seconds, while the manual attendance method averaged about 17.8 seconds of the execution time. Thus, the biometrically based attendance process findings show improved performance compared to the manual attendance method.

Adewole et al, collectively proposed a fingerprint biometric attendance system for non-academic employees of tertiary institutions [8]. With the aid of fingerprint recognition software, the device gathered attendance remotely and stored all collected data for future operations accessibility. The company's biometric attendance system used an automated system to monitor employee attendance and thus allow additional monthly attendance projections, minimizing human error.

Swyfttapp, an NFC-based attendance system with fingerprint authentication enablement, was suggested by Kommey, Anyane-Lah, and Amuzu [9]. The researchers addressed the project using NFC software in combination with fingerprint authentication technology. The research work was designed to work with the API and the web-based framework, which introduced a more practical approach to tracking and controlling university attendances. The researchers concluded that the project was designed to address the need for a faster and more effective way of monitoring student attendance and to provide a platform for the management and analysis of data collected from such attendance at the university.

Hualin, Qiqi, and Yujing worked on a fingerprint attendance machine that was design based on a C51 singlechip microcomputer [10]. The fingerprint attendance system, marked with the fingerprint authentication model, eliminated the fingerprint attendance process failure from any unregistered person's fingerprint. Thus, it essentially put an end to attendance management human factors by fully embodying the fairness of attendance management and avoiding unnecessary staff conflicts. The system used the STC89C52 microcontroller as a central control chip, 12864 LCD as a man-machine interface, a matrix keyboard as an input student ID, and a sensor identification module.

Adal et al. proposed an innovative attendance vigilance framework that used an android platform controlled using a wireless network and an automated combination of biometric fingerprint authentication [11]. The authors made use of an innovative attendance program that used a mobile ID card that was generated using an iOS phone. The authors built a Smartphone application in Java which guaranteed individual employee's presence using biometric fingerprint authentication. The entire workplace had a Wi-Fi zone. As a result, the connection/disconnection of the android device to the router was programmed to determine the workplace's availability status.

Automatic Door Unit Radio Frequency Identification (RFID) Based Attendance System was proposed by Nwaji, Onyebuchi, and Kelechi [12]. The hardware and software were the two critical components of the device. The processing unit, a serial to USB converter cable connecting the RFID reader, a low-frequency reader, and the RFID reader make up the hardware. In addition, visual Basic.Net was used to create the Time Attendance System GUI (Graphic User Interface), and the GUI was used to access the attendance system.

A system that provides accurate and efficient attendance management service to staff and students that had access to the portal system was presented by Badejo et al. [13]. They developed an interactive database system to simplify attendance management and evaluated fifty students in five experiments. The $98 \%$ accuracy obtained by the program suggested the viability of large-scale deployment and interoperability of multiple devices that existed.

Singh, Jain, and Jain analyzed a literature survey on fingerprint recognition using the level 3 feature extraction 
method [14]. The SIFT algorithm was used to design and balance the Level 3 fingerprint feature extraction process in their survey. High-quality fingerprints were captured using an optical scanner, and the image was normalized using Gaussian blurring and sliding window contrast adjustment. Pores were being collected, and their respective sizes were calculated. The SIFT algorithm allowed the approximation and storage of the pores to fit using the database prototype. SIFT (Scale-Invariant Features Transform) is a computer vision algorithm for recognizing and defining local features in images.

The implementation of a fingerprint-based biometric attendance system was proposed by Ahmed et al [15]. The research work implemented the use of a microcontroller to implement the fingerprint attendance system. The microcontroller controlled the entire process and displayed the different instructions via LCD. The printing of the index served as a signature for the device entry. The system can select the institution's departure time, which in turn helped the transportation department in scheduling buses. The findings showed that the automated attendance management system performed better than the conventional manual system.

Shoewu et al, designed an RFID-based automated attendance system for students (R-BASS). The authors created the R-BASS system to monitor student attendance using the RFID sensors installed in classrooms [16]. In addition, the design noted that one could insert the RFID tag into the individual's ID card based on the confirmation of student identification during the entrance process.

\section{MethodOLOGY}

Since the system consists of both software and hardware elements, it is classified as an embedded system. The hardware components include an RFID reader, a fingerprint sensor unit, an Arduino Mega, an LCD, a buzzer, and a 12V power supply. Therefore, the project aims to achieve a specific purpose and outcomes, not to remain isolated from the project's objectives. Several steps were taken in developing this project and can be summarised thus:

- Collection of information.

- Information collection using the respective problemsolving choice approaches.

- Selection of construction tools and availability of materials.

- Construction of hardware.

- The microcontroller programming selection based on the availability of its shielding.

- Integration of technology and hardware for testing.

The RFID reader and the fingerprint scanner are connected to the microprocessor. The data (ID numbers and fingerprint images) are registered as a part of the program into the Arduino Mega to identify each user automatically. A $12 \mathrm{~V}$ power supply will power the fingerprint sensor module, RFID scanner, memory card module, RTC module, and Arduino Mega of the system, as shown in Fig. 1. Attendance is being taken only when both input data match the previously stored data.

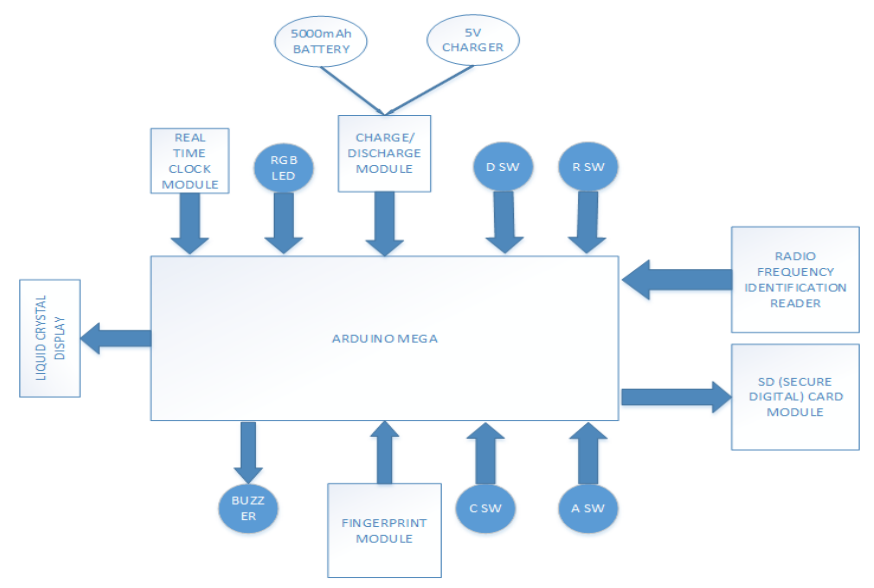

Fig. 1. Block Diagram of the system.

The design includes shield making, connecting the power source, and supplying the proper voltage to the system, programming of the RTC module, fingerprint, RFID module, and SD card module, and linking them to make an embedded system. The circuit diagram is being shown in Fig. 2, and it was simulated with Fritzing.

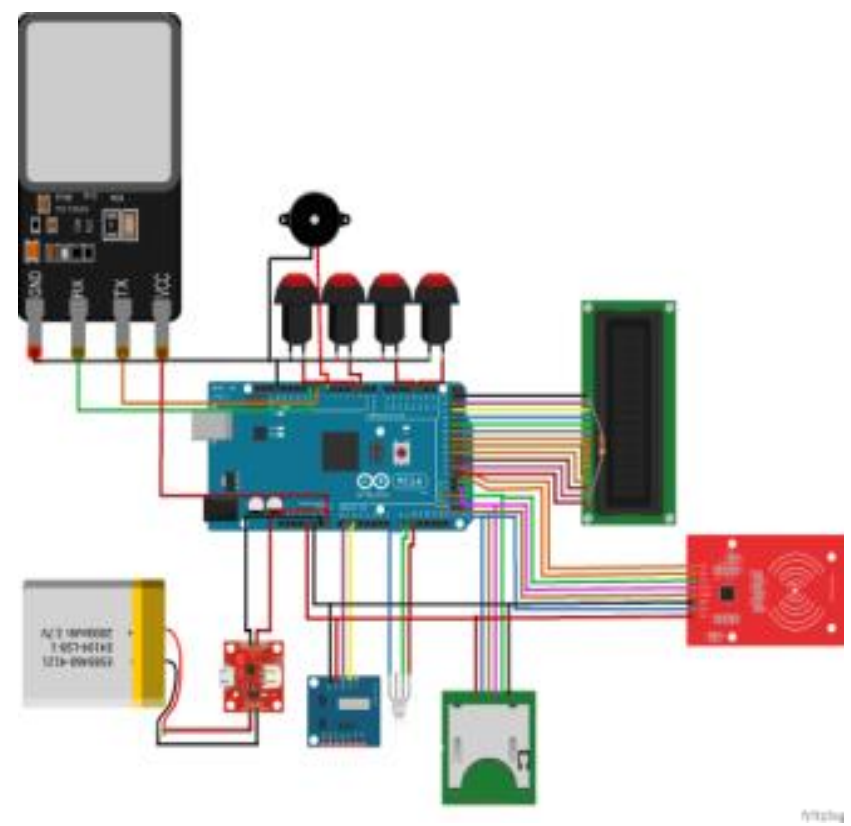

Fig. 2. Circuit Diagram of the system.

The standard simulation was not done on this project because there is no library for the fingerprint and RFID on Proteus, Fritzing, Mutism, and other standard simulation tools. As a result, in the loop simulation, HIL (hardware-inthe-loop) was implemented. HIL is a technique for developing and testing complex real-time embedded systems. HIL simulation provides an active structure by transferring the control plant's complexity to the test platform [17]. With the aid of the shield, it was easy to build and test the system. The shield is being shown in Fig. 3. 


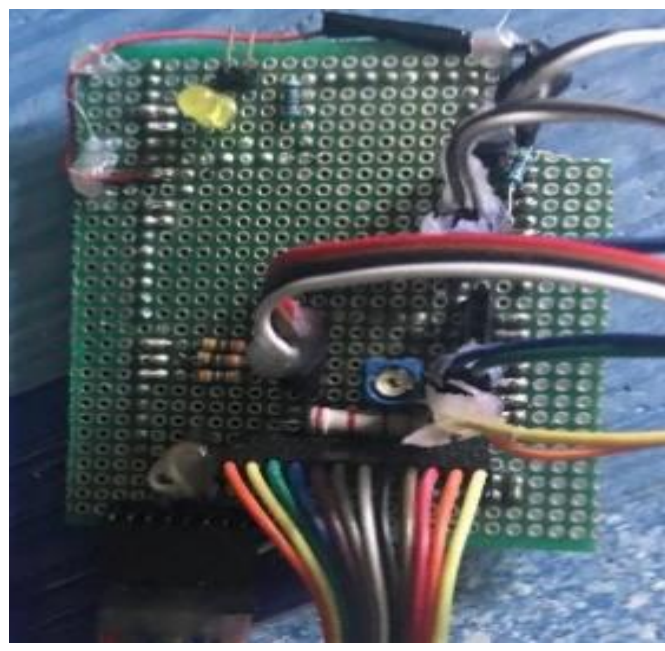

Fig. 3. Front view of the shield made.

A double-sided Vero board was used to make the total system circuit shield. The shield consists of all the ports needed to make it easy to plug and remove other modules from the Arduino Mega microcontroller. All the modules were tested individually using the shield and the microcontroller. After trying all individually, then they were all assembled and coupled unto the shield. It makes it easier to connect them and is less error-prone than to start joining a wire to a Vero board.

- A lithium-ion battery of $5000 \mathrm{mAh}$ is being used to power the system. The battery energy storage has a nominal voltage of $3.7 \mathrm{~V}$ and a total voltage of $4.2 \mathrm{~V}$. It was then connected to a charge/discharge module which is built explicitly for lithium-ion batteries. This module does allow charging lithium-ion batteries with a $5 \mathrm{~V}$ USB charger. A switch was connected to the power source to ON/ OFF the total system. A LED indicator was connected to show when the system is ON.

- $\quad$ RTC uses the $\mathrm{I}^{2} \mathrm{C}$ communication protocol, and this was connected to pin 16 and 17, which are the SDA (Serial Data) and SCK (Serial Clock) on the Arduino Mega. The communication pins were connected to pin 10 and 11 on the Arduino Mega. Pin 10 and 121 are not dedicated pins for serial communication on Arduino Mega. The Arduino Mega microcontroller used the main ports for serial communication to communicate with the personal computer (PC). So, 10 and 11 were used because there is a library that does allow the use of non-dedicated serial communication pins for serial communication. The name of the library is "SoftwareSerial.h".

- The LCD has 16 pins, but a total of 12 pins were used. The first two pins were used for the power supply (+ve and GND). The next pin was used for contrast, which is connected to the variable resistor. The next pin is the register select pin which was used to switch from one selected to another. The next is the read and write pin, which was connected to the GND for reading the only purpose. And the last out of the first six pins, which are Enable (E) pins, were combined so that the LCD could latch information presented in the data pins. The next set of 6 pins includes: pin D4 to D7 to carry data and the LED +ve and LED -ve pins. A $220 \Omega$ resistor was connected to the LED +ve and LED - ve pins to limit the backlight's current.
- The RFID module was first tested alone with the shield, the Arduino Mega microcontroller, and the MR55 library. This library was integrated into the Arduino IDE, which is in turn used to program the module. The radio frequency identification reader uses the SPI communication protocol for communicating with Arduino Mega. It was connected to the SD card module because the SD card module also uses the SPI communication protocol. The RFID reader has six pins, and out of the 6, 2 are outputting their contents onto the Arduino Mega microcontroller. The other four were connected with the SD card module before joining it to the Arduino Mega. The connection is possible because SPI does allow communication with more than one SPI device by using the chip select pin for the device.

- The Secure Digital card module also uses the SPI communication protocol for communicating with Arduino Mega. It also has six pins, and out of the 6, 2 is outputting their contents onto the Arduino Mega Microcontroller. The other four were connected with the RFID module before joining it to the Arduino Mega. The whole module was then attached to make an embedded attendance system, as shown below.

\section{TEST AND DATA}

\section{A. Enrolment Mode Testing}

The testing steps are explained as follows:

- On entering the enrolment field, the admin will have to check, and this is because the administrator only has the right to enroll a student. The admin can be either a lecture or the administration. The admin can merely do the check by merely placing their registered finger on the fingerprint scanner.

- Next is informing the student to activate the enrolment mode on his or her RFID card. Since the micro-controller has the card's UID code, the corresponding no is verified. The system then displays the fingerprint ID no on the card and then knows the fingerprint slot no.

- The program prompts the student to insert a fingerprint. The system will then take the picture when the finger is put on the scanner.

- The system will then prompt the student to place the finger again to confirm whether it matches. If the fingerprint scans images match, the system will store the image on the array.

The above steps were repeated for the other nine students. Fig. 4 shows the steps in testing the enrolment mode.

\section{B. Attendance Mode Testing}

Attendance mode is the first mode, and before using it, it does not need administration authorization. Testing steps:

- When a student inserts his or her card into the system, the RFID reader scans the code and looks up the student's number.

- The system then asks the student to place his/ her finger to confirm if it is indeed him/her.

- After confirmation, the system then marks the particular student present and then stores it on the array created for attendance. 


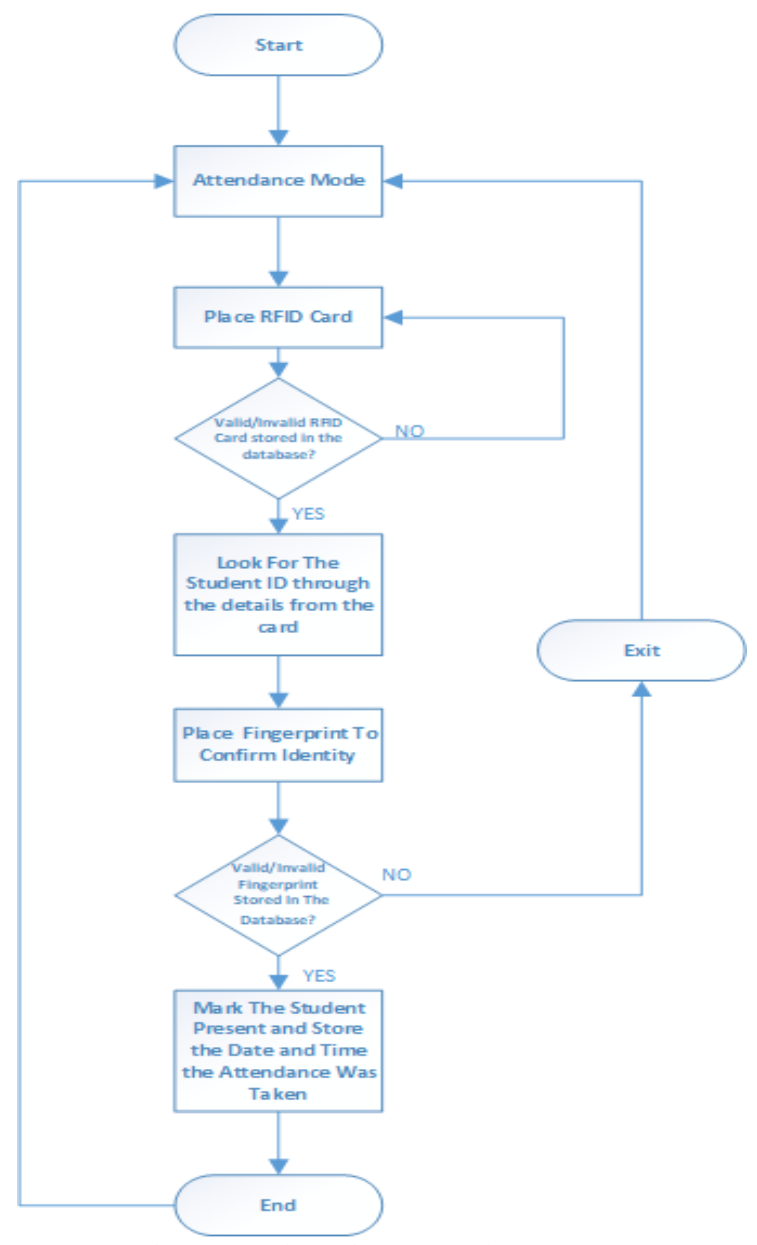

Fig. 4. Steps in testing the enrolment mode.

\section{Compilement mode testing}

Compilement mode helps put together all the present students. Immediately the compilement mode has been activated; one can no longer adjust the attendance. Testing steps:

- The admin is required to verify before compiling the attendance. When the admin fingerprint is being placed and verification has been done, the system then autogenerates the attendance.

- The generated attendance is then stored on the SD card in the form of a TXT file. The generated attendance does show the ID no, date, and time together with status.

\section{Data}

In functional requirements, the system was tested by different users. Most of the users were registered already by the admin using the enrolment steps, while one of the students was not registered. After analyzing ten students with no five users, the result is shown in Table I.

TABLE I: THE RESULT FROM THE TEST

\begin{tabular}{cccc}
\multicolumn{4}{c}{ TABLE I: THE RESULT FROM THE TEST } \\
\hline No & Date & Time & Status \\
\hline 1 & $12-21-2019$ & $10: 23: 32$ & MARK \\
2 & $12-21-2019$ & $10: 24: 05$ & MARK \\
3 & $12-21-2019$ & $10: 24: 26$ & MARK \\
4 & $12-21-2019$ & $10: 24: 51$ & MARK \\
5 & & & \\
6 & $12-21-2019$ & $10: 25: 17$ & MARK \\
7 & $12-21-2019$ & $10: 25: 46$ & MARK \\
8 & $12-21-2019$ & $10: 26: 12$ & MARK \\
9 & $12-21-2019$ & $10: 26: 35$ & MARK \\
10 & $12-21-2019$ & $10: 27: 08$ & MARK \\
\hline
\end{tabular}

\section{RESUlT}

Effectively, designing and implementing this project has created a long-lasting solution to some of the various problems encountered when taking part in institutions. The vast rise in the advancement and bypassing of technology can make this project forged and hacked, making the project inefficient. This study is relevant because it responds to specific questions related to the construction of an attendance system. Some challenges were encountered during the implementation and construction stages.

\section{CONCLUSION}

The manual method of taking attendance, which involves pen and paper records, has been used as the conventional way of taking attendance. Implementing an electronic attendance management system has been proven to help save time, secure attendance data and privacy, and help save time. The RFID-biometric attendance system gives administrators quick access to student attendance data and allows them to keep track of their students. The system is dependable, safe, and effective, and it can replace the outdated and inefficient manual attendance management process. Conclusively, developing a hybrid attendance system (Radio-frequency Identification and Biometric Security) that uses students to investigate was constructed. A system model was also developed, which included coordinated software and hardware design (embedded system). The design offered a convenient and safe attendance method compared to the conventional attendance system in reading time. The data was better-structured thanks to the external memory. The fingerprint data header configuration provides high security and high-speed performance and makes it easier to access student information with an RFID card. The conventional ways of attending and attending in a short time have always been needed to be removed; this RFID-Biometric attendance system can be happy to cater for it.

\section{REFERENCES}

[1] V. Kassarnig, A. Bjerre-Nielsen, E. Mones, S. Lehmann, and D. D. Lassen, "Class attendance, peer similarity, and academic performance in a large field study," arXiv, 2017.

[2] K. Mohammed, A. S. Tolba, and M. Elmogy, "Multimodal student attendance management system (MSAMS)," Ain Shams Eng. J., vol. 9 , no. 4, pp. 2917-2929, 2018, DOI: 10.1016/j.asej.2018.08.002.

[3] H. C. Paul, D. N. Monday, and S. Elango, "Biometric and Rfid Technology Fusion: a Security and Monitoring Measures To Enhance, "Int. J. Inf. Syst. Eng. IJISE, vol. 4, no. 1, pp. 27-35, 2016.

[4] O.G. Chiagozie and O.G. Nwaji, "Radio Frequency Identification (Rfid) Based Attendance System with Automatic Door Unit," Academic Research International, vol. 2, no. 2, pp. 168-183, 2012.

[5] MK Sabri, MZ. Abdul-Aziz, M.S. Shah and M.F. Abd-Kadir, "Smart Attendance System by Using RFID, "2007 Asia-Pacific Conference on Applied Electromagnetics proceedings, pp. 1-4, 2007.

[6] PU Eze, C.K. Joe-Uzuegbu, U. Laz and FK Opara, "Biometric-based Attendance System with Remote Realtime Monitoring for Tertiary Institutions in Developing Countries," IEEE NIGERCON 2013, pp. 1 8,2013

[7] O. Shoewu, N.T. Makanjuola and S.O. Olatinwo, "Biometric-based Attendance System: LASU Epe Campus as Case Study," American Journal of Computing Research Repository, vol. 2, no. 1, pp. 8-14 2014.

[8] KS. Adewole, S.O. Abdulsalam, R.S. Babatunde, T.M. Shittu, and M.O. Oloyede, "Development of Fingerprint Biometric Attendance System for Non-Academic Staff in a Tertiary Institution, "Computer Engineering and Intelligent Systems, vol. 5, no. 2, pp. 62-70, 2014. 
[9] B. Kommey, O. Anyane-Lah, and W.E. Amuzu, "SwyftTapp: An NFC Based Attendance System Using Fingerprint Authentication," International Journal of Engineering, Science and Technology, vol. 10 , no. 1, pp. 23-39, 2018.

[10] Z. Hualin, W. Qiqi, and H. Yujing, "Fingerprint Attendance Machine Design Based on C51 Single-chip Microcomputer, "International Conference on Computer Technology, Electronics and Communication (ICCTEC), pp. 774-777, 2017.

[11] H. Adal, N. Promys, S. Srabanti, and M. Rahma, "Android Based Advanced Attendance Vigilance System Using Wireless Network with Fusion of Bio-metric Fingerprint Authentication," International Conference on Advanced Communications Technology (ICACT), pp. 217-222, 2018

[12] O.G. Nwaji, N.C. Onyebuchi and O.F. Kelechi, "Automatic Door Unit Radio Frequency Identification (RFID) Based Attendance System,' International Journal of Science and Emerging Technology, vol. 5 , no.6, pp. 200-211, 2013.

[13] J.A. Badejo, C. C. Eke, S. I. Popoola, T. O. Odu, A.A. Atayero, "Integrating Automated Fingerprint-based Attendance into a University Portal System," 2017 International Conference on Computational Science and Computational Intelligence, pp. 10161020, 2017.

[14] C.P. Singh, S. Jain, and A. Jain, "Literature Survey on Fingerprint Recognition Using Level 3 Feature Extraction Method, "International Journal of Engineering and Computer Science, vol. 3, no. 1, pp. $3805-$ 3812, 2014.

[15] N. Ahmed, I. Hossain, R.H. Rafi, H. Rashid, Q. Nawaz, and S.T. Reza, "Design \& Development of Fingerprint Based Biometric Attendance System, "International Conference on Computer, Communication, Chemical, Materials and Electronic Engineering, pp. 156-159, 2007.

[16] O. Shoewu, N.T. Makanjuola, A.A. Ajasa, and O.J. Ayangbekun, "Design and Implementation of an RFID Based Automated Students Attendance System," Journal of Advancement in Engineering and Technology, vol. 3, no. 2, pp. 1-6, 2015.

[17] A.V. Tumasov, A.S. Vashurin*, Y.P.Trusov, E.I. Toropov, P.S Moshkov, V.S. Kryaskov, and AS Vasilyev, "The application of hardware-in-the-loop (HIL) simulation for evaluation of active safety of vehicles equipped with electronic stability control (ESC) systems,' Procedia Comput. Sci., vol. 150, pp. 309-315, 2019, DOI: 10.1016/j.procs.2019.02.057

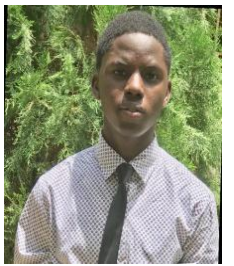

E. O. Badmus was born in Nigeria in the year 2000 He obtained his first degree in Electrical Electronics Department from Federal University Oye-Ekiti, Ekiti State, Nigeria, in 2020.

He has previously worked as an Engineering Intern in Osun State Broadcasting Station, which houses significant television and radio stations between 2018 and 2019. He equally served as an Electrical Engineer Graduate Intern in Pacific Energy Company Limited, Olorunsogo Power Plant, Phase I, Ogun State, Nigeria. He is also a member of the Nigeria Society of Engineers (NSE).

Smart Sensing, Energy Storage, Renewable Energy, Smart, and Microgrids are some of Emmanuel's research interests. He is presently concentrating on graduate school applications for the academic year 20212022.

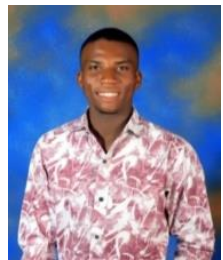

O. P. Odekunle was born into the family of Dr and Mrs Olusogo Odekunle of Osu, Osun State, Nigeria. He bagged his Bachelor of Engineering degree with a Second Class Upper Honours in Electrical and Electronics Engineering from the Federal University Oye-Ekiti in February 2020.

He had his Undergraduate Internship at the British American Tobacco, Ibadan, between July 2018 and January 2019. He equally had his Graduate Engineering Internship at the Department of Engineering works at the Cocoa Research Institute of Nigeria between March 2020 and March 2021. He is a Graduate Member of the Nigerian Society of Engineers as well as the International Association of Engineers.

Oluwamurewa is currently focusing on the application for MSc in the Fall 2022 Academic Session. His research interests are in Home Automation, Microgrids, Smartgrids, Renewable Energy Systems, Power Systems, Control Systems, and Smart Sensing.

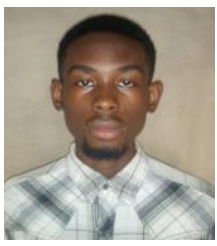

D. O. Oyewobi was born and brought up in the southwestern region of Nigeria. He obtained his Bachelor of Engineering degree from the Department of Electrical and Electronics Engineering at the FUOYE, Nigeria, in February 2020.

He had his undergraduate internship at the Transmission Company of Nigeria within the years of 2018 and 2019 During his one service year to his country, he served as a graduate engineering intern at the engineering department of Westmidlands Communications located in Ibadan, Nigeria. He's also an expert in computer hardware repairs and a data analyst. His research interests include Data Analytics, Renewable Energy, Home Automation, Power Systems, and Smart Sensing. 